\title{
Efficient Spectrum Sensing for the Relay Based Cognitive Radio Network for Enhancing the Network Coverage for Wireless Patient Monitoring System
}

\author{
Geoffrey Eappen \\ School of Electronics Engineering \\ Vellore Institute of Technology \\ Vellore, India \\ Email:geofz121@gmail.com
}

\author{
Shankar T \\ School of Electronics Engineering \\ Vellore Institute of Technology \\ Vellore, India \\ Email: tshankar@vit.ac.in
}

\author{
Rajagopal Nilavalan \\ Electronics and Computer Engineering \\ Brunel University \\ London, U.K. \\ Email:Nila.Nilavalan@brunel.ac.uk
}

\begin{abstract}
The wireless Patient Monitoring System (PMS) is facing the issue of spectrum congestion. The $5 \mathrm{G}$ technology based wireless PMS is a promising solution. With the advancement in the wireless telecommunication technology from 4G to $5 \mathrm{G}$, the important paradigm to be considered for the state of the art technique for the 5G is the device to device (D2D) communication. One of the important issue associated with the advanced wireless communication technology is the network disconnection due to network blockage. Moreover, inefficient usage of the spectrum is the another major concern in wireless communication services. In this paper, by efficiently utilizing the unused spectrum the Cognitive Radio (CR) Relay assisted cooperative D2D communication has been aimed to enhance the network capacity (data rate) of the User Equipment (UE)/Licensed User (LU) which is in the network blockage area for wireless remote PMS. The CR enabled with Expert Whale Optimization Algorithm (EWOA) has been proposed for efficient spectrum sensing. The CR based relay technique for D2D communication with UE can significantly improve the performance of the network capacity without any investment on deploying new base stations. Simulation results exhibits that the performance of the network with EWOA-CR based relay technique has significantly improved.
\end{abstract}

Keywords-Cognitive Radio Network; Whale Optimization Algorithm; Spectrum sensing; Device 2 Device Communication

\section{INTRODUCTION}

In the current scenario, the wireless PMS can play a major role in improving the well being of a population. The wireless PMS gives more flexibility and mobility to the patients. In human diseases takes certain time period to develop. During the period human body vitals fluctuates. A continuous patient monitoring can help in predicting the early signs of diseases via patient body vital data. But a seamless connection is required between patient and the supervisors ( Doctors, Nurses etc.). Such seamless connection without affecting the mobility of the patients and operating remotely in any condition calls for the need of wireless PMS. But the wireless cellular network is facing the problem of the capacity bottleneck. Moreover, mmWave (Millimeter Wave) frequency does not have much penetration ability. Such a scenario results in a network blockage condition. In $4 \mathrm{G}$, to overcome such problems techniques like carrier aggregation and Multiple Input Multiple Output (MIMO) antenna scheme was deployed [1]. Turbo coding and Space time block coding schemes were employed to enhance the channel capacity [2-4] via multipath wireless communication. These schemes could not completely solve the problem of network capacity as channel capacity can not be increased infinitely by incorporating multiple antennae which further results in additional hardware costs. With the D2D link, it is possible to increase network capacity without additional cost on network infrastructure. A D2D link is a key enabler of 5G wireless communication technology [21] and the release 12 of the Long Term Evolution (LTE) from 3GPP is its clear proof [22]. The 3GPP release 12 of LTE has also mentioned about the D2D communication for public-safety and proximity based communication and services [24] [25]. The D2D communication can be employed for enhancing the data rates, decreasing the power consumption, decreasing the network latency and providing different application specific services [23]. In addition to that D2D communication also enables offloading of the cellular network, communication between vehicle to vehicle, and low latency applications [26] [27]. The UE-UE based relay system is studied in [5] in which a UE acts as a relay to the other UEs which are in network blockage area with the aim of enhancing the network capacity without additional cost for the operator for establishing relay BS infrastructure [28]. The drawback associated with the UE-UE communication is that the UE relay is not stationary and there are chances that UE relay can itself get into some network blockage condition. With the increasing demands in the D2D based application can result in the network congestion, spectrum scarcity which can cause reduced data rate and enhanced latency [29] [31].

To overcome such problems a CR based relay system is proposed to establish link from UE to the Base Station (BS) corresponding to the UE and vice versa, throughout the paper the base station corresponding to the UE is denoted as BS and the base station corresponding to the $\mathrm{LU}$ is denoted as $L U_{B S}$. The $\mathrm{CR}$ based relay network can potentially proved as effective in overcoming the fading problem in $\mathrm{D} 2 \mathrm{D}$ communication by providing high chan- 
nel capacity with low cost [30] [31]. A CR network can dynamically adapt and allocate different spectrum resources to the cognitive radio users/ secondary users via efficient spectrum sensing and sharing [6]. The CR based relay scheme is effectively employed in [32] for cooperative D2D communication. Authors in [33] implemented CR based relay network with cooperative communication aided Secondary Users (SUs) are served by the base station. In the above mentioned works [30-33], the CR for relay network employed conventional energy detection technique for the spectrum sensing. The spectrum sensing is the most important paradigm of the CR network. An effective spectrum sensing is very crucial for efficient detect detection of spectrum holes. Inefficient spectrum sensing can cause interference and can also results in low opportunistic throughput. The conventional energy detection techniques is the least complex but suffers at low SNR [6]. Therefore, in this paper an effective CR based relay network is implemented for overcoming network blockage issue with the efficient spectrum sensing technique. The spectrum sensing employed in this CR system is the weighted cooperative spectrum sensing scheme in which the weight values are optimized using novel EWOA, thus guarantying an efficient spectrum hole detection. The proposed scheme is compared with the existing Particle Swarm Optimization (PSO) and Whale Optimization Algorithm (WOA) based CR network. The PSO and its modified version is extensively employed for spectrum sensing [34] [35] [36] [37]. The prime drawback associated with PSO is that it is capable of finding very good solution but it converges before obtaining global optimum solution [38]. For spectrum sensing it is very important that the algorithm do not converge to the near global solution but should obtain Global optimum solution so as to avoid interference with the PU and to carryout the remaining process of a CR network i.e opportunistic data transmission, spectrum sharing, spectrum mobility more effectively.

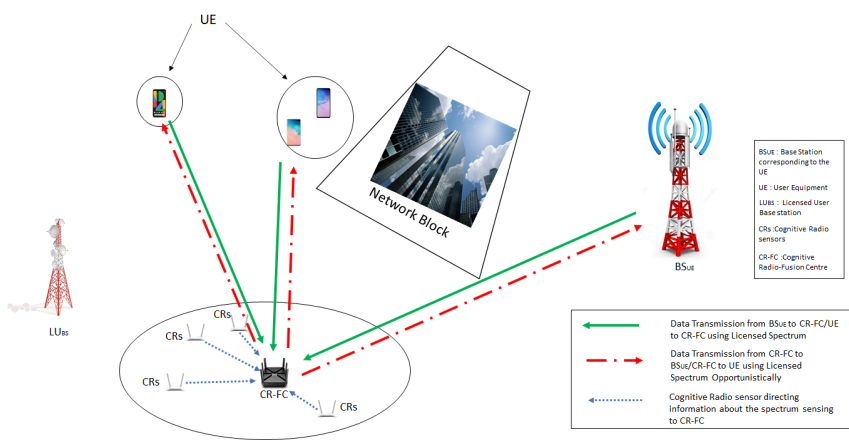

Figure 1: Flow diagram of the proposed model

From Figure 1 it can be illustrated that when a UE enters the network blockage region the proper link between BS and UE does not exist and can affect its network performance. The deployed Cognitive Radio-Fusion Center (CR-FC) as- sisted with Cognitive Radio sensors (CRs) acting as a relay between UE and BS, UE and UE can significantly improve the network performance. In this scenario, BS has prior knowledge of the nearby CR-FC in a particular geographical location and CR-FC is able to establish a proper link with the BS. In the case of network blockage during downlink, the BS communicates to CR-FC and CR-FC with the assistance of CRs performs spectrum sensing to detects the presence and the absence of the Licensed User (LU)/Primary User (PU) of a particular geographical location. The vacant spectrum is detected and data is transferred to UE by opportunistically accessing the licensed spectrum holes. Here th LU corresponds to TV broadcast system and CR-FC is employed to detect the TV white spaces. The UE gets the information of the CR-FC and transmit data back to the CR-FC via the licensed spectrum. The CR-FC again opportunistically access the licensed spectrum holes and transmits data back to the BS, thus establishing BS-UE connection. Similar process is carried out by the CR-FC to establish UE-UE connection within the same network blockage region. The CR-FC can also communicate with the other CF-FCs deployed in the specified region to have better network coverage.

The proposed spectrum sensing scheme is performed in a weighted cooperative manner, where each CRs performs spectrum sensing and transmits information to the CR-FC via weights. The CR-FC performs weight optimization using MWOA and efficiently detects the vacant spectrum.

\section{Mathematical Modeling of the Proposed Spectrum Sensing Scheme}

In this paper, throughput is analyzed for the data transmission from BS (Corresponding to the UE) to UE via CR$\mathrm{FC}$, and UE to BS via CR-FC. In order to have generic problem following considerations have been made: Let $j$ be the number of UE in the network blockage area located in a 2D plane, $k=1,2,3 \ldots M$ denotes the orthogonal subcarrier each having bandwidth $=W$. These subcarriers are available in CR-FC and BS for resource allocation. There are $l=1,2,3, \ldots . C R_{s}$ number of CRs and 1 CR-FC forming the relay for assisting data transmission between BS to UE and UE to BS.

Channel gain between BS and CR-FC for the transmission meant for the $j^{t h}$ UE over $k^{\text {th }}$ subcarrier at time slot $t$ can be written as $h_{j, k, t}^{B, C R F C}$. Similarly channel gain between CRFC and $j^{t h}$ UE over $k^{t h}$ subcarrier at time slot $t$ can be denoted as $h_{j, k, t}^{C R F C, U E}$. The transmission power from BS to CR-FC for the $j^{\text {th }}$ UE over $k^{\text {th }}$ subcarrier and at time slot $t$ can be written as $T p_{j, k, t}^{B, C R F C}$. The CR-FC transmission power is fixed value for any subcarrier and it is denoted as $C R_{p}$. It is assumed that each CR-FC based relay is having unit buffer size, i.e. if BS to CR-FC transmission occurs at $t^{\text {th }}$ time slot then CR-FC to UE transmission occurs at $(t+1)^{t h}$ time slot. In the weighted cooperative spectrum sensing scheme while the CR-FC makes the final decision 
and performs data transmission, the CRs parallely performs spectrum sensing so that the buffer size would be unity.

The BS power received at CR-FC can be estimated as Eq.1:

$$
T p_{j, k, t}^{B, C R F C, r}=T p_{j, k, t}^{B, C R F C} \times\left\{\frac{4 \pi f_{c} d_{o}}{c}\right\}^{-2} \times d_{B S}^{-n^{\prime}} / N_{b s}
$$

here, $f_{c}$ is the licensed carrier frequency associated with the $\mathrm{BS}, d_{o}$ is a reference distance, $d_{B S}$ is the distance between BS and the CR-FC calculated

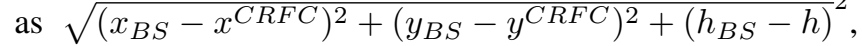
$\left(x_{B S}, y_{B S}, h_{B S}\right)$ is the coordinates associated with the BS location, $n^{\prime}$ is the path loss exponent, $N_{b s}$ is the average number of users associated with the BS.

The transmission power $C R_{p}$ of CR-FC at some height $h$ as shown in Figure 2 is different from the CR-FC power received at $\mathrm{UE} C R_{p}^{r}$. The received CR-FC power at $\mathrm{UE}$ is derived as under:

The coordinates of UE $j \in \ell$ is $\left(x_{j}, y_{j}\right)$ and the coordinates

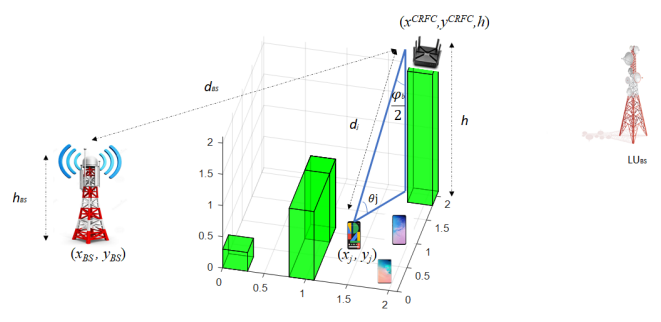

Figure 2: Detailed representation of the proposed model

of CR-FC is $\left(x^{C R F C}, y^{C R F C}, h\right)$. For the communication link between CR-FC and UEs, the CR-FC with directional antenna with half beamwidth $\varphi_{b}$. The CR-FC antenna gain can be denoted as [13]:

$$
g_{a}=\left\{\begin{array}{cc}
g_{a}^{3 d B} & \frac{-\varphi_{b}}{2} \leq \psi \leq \frac{\varphi_{b}}{2} \\
g_{a}(\psi) & \text { otherwise }
\end{array}\right.
$$

here $\psi$ is the sector angle, $g_{a}^{3 d B} \approx \frac{29000}{\varphi_{b}^{2}}, \varphi_{b}$ in degree is the gain of the main lobe [14], $g_{a}(\psi)$ is the gain outside the main lobe. Because of the uncertainity associated with the UE location, height and the obstacles, it is important to consider the randomness in the link between CR-FC and UE and also vice versa. Therefore, the link between CR-FC and UE can be of Line of Sight (LoS) and Non Line of Sight (NLoS) with certain probabilities [16].

As per the 3GPP 3D model of the Urban Macro scenario for the UE of height $1.5 \mathrm{~m}$ [15], the LoS probability between CR-FC and UE can be modelled as [16] [18] [20]:

$$
\operatorname{Pr}^{\operatorname{LoS}}=\frac{1}{1+\alpha \times \exp \left(-\beta\left|\theta_{j}-\alpha\right|\right)}
$$

here $\alpha, \beta$ are the constants corresponding to carrier frequency and the environment type like rural, urban, and urban dense, $\theta_{j}=\frac{180}{\pi} \times \sin ^{-1}\left(\frac{h}{d_{j}}\right), d_{j}$ is the distance between CR-FC and UE, calculated as $\sqrt{\left(x_{j}-x^{C R F C}\right)^{2}+\left(y_{j}-y^{C R F C}\right)^{2}+h^{2}}$.

The NLoS probability can be written as Eq.(4):

$$
\operatorname{Pr}^{N L O S}=1-\operatorname{Pr}^{L o S}
$$

The coverage of directional antenna with beamwidth $\varphi_{b}$ is given as $r_{a}=h \times \tan \left(\frac{\varphi_{b}}{2}\right)$ [17].

For the transmission power of $C R_{p}$, the received power of CR-FC at $j^{\text {th }}$ UE for the LoS and NLoS link is given as in Eq.(5):

$$
C R_{p}^{r}=\left\{\begin{array}{cl}
C R_{p}+g_{a}^{3 d B}-L o_{d B}^{U E}-E_{L o S}, & \text { LoS Link } \\
C R_{p}+g_{a}^{3 d B}-L o_{d B}^{U E}-E_{N L o S}, & \text { NLoS Link }
\end{array}\right.
$$

For a fixed location of CR-FC and varying locations of the UEs the path loss associated with this situation is to be considered averaged path loss. Therefore, The path loss $L o_{d B}^{U E}$ between CR-FC and UE is calculated as in Eq.(6):

$L o_{d B}^{U E}=P r^{L o S} \Upsilon_{1}\left\{\frac{4 \pi f_{s}^{c} d_{j}}{c}\right\}^{n^{\prime}}+\operatorname{Pr}^{N L o S} \Upsilon_{2}\left\{\frac{4 \pi f_{s}^{c} d_{j}}{c}\right\}^{n^{\prime}}$

where, $f_{s}^{c}$ is the spectrum sensed carrier frequency of the CR-FC, $n^{\prime}$ is the path loss exponent, $d_{i}$ is the distance between CR-FC and UE and $c$ is the speed of light, $\Upsilon_{1}, \Upsilon_{2}$ are the coefficients of the excessive path loss during LoS and NLoS conditions respectively.

The shadow fading in $\mathrm{dB}$ for the LoS and NLoS link is denoted as $E_{L o S}$ and $E_{N L o S}$ respectively. The $E_{L o S}$ and $E_{N L o S}$ has the normal distribution as $E_{L o S} \sim \mathbb{N}\left(\mu_{L o S}, \sigma_{L o S}^{2}\right), \quad E_{N L o S} \sim \mathbb{N}\left(\mu_{N L o S}, \sigma_{N L o S}^{2}\right)$ respectively. The $\sigma_{L o S}, \sigma_{N L O S}$ depends on environmental condition and the elevation angle as denoted in Eqs.(7) and (8) [18]:

$$
\begin{gathered}
\sigma_{L o S}=J_{1} e^{\left(-J_{2} \theta_{j}\right)}, \\
\sigma_{N L O S}=G_{1} e^{\left(-G_{2} \theta_{j}\right)}
\end{gathered}
$$

where, $J_{1}, J_{2}, G_{1}, G_{2}$ are the constants depending on environmental constraints.

The received $j^{\text {th }}$ UE power at CR-FC is calculated as in Eq.(9):

$T p_{j, k}^{U E, C R F C, r}=\left\{\begin{array}{cl}T p_{j, k}^{U E, C R F C}-L o_{d B}^{U E}-E_{L o S}, & \text { LoS Link } \\ T p_{j, k}^{U E, C R F C}-L o_{d B}^{U E}-E_{N L o S}, & \text { NLoS Link }\end{array}\right.$

The link between CR-FC and BS can be modeled as in Eq.(10), the received CR-FC power at $\mathrm{BS}$ is:

$$
C R_{p, B S}^{r}=C R_{p} \times\left\{\frac{4 \pi f_{c} d_{o}}{c}\right\}^{2} \times d_{B S}^{-n^{\prime}}
$$

The achievable throughput $T_{j, k}^{h, B-U E}$ for the transmission from the BS to the $j^{\text {th }}$ UE over the $k^{\text {th }}$ subcarrier can be evaluated as in Eq.(11)[5][7]:

$$
T_{j, k}^{h, B-U E}=\frac{1}{2} \log _{2}\left\{1+\frac{T p_{j, k, t}^{B, C R F C, r} \times C R_{p}^{r} \times h_{j, k, t}^{B, C R F C} \times h_{j, k, t}^{C R F C, U E}}{\left(N_{0} W\right) \times\left(T p_{j, k, t}^{B, C R F C, r} \times h_{j, k, t}^{B, C R F C}+C R_{p}^{r} \times h_{j, k, t}^{C R F C, U E}\right)}\right\}
$$


here $N_{0}$ is the noise power spectral density. As time

index considered is of unit buffer size, so time index can be omitted. Therefore Eq.(11) can be re-written as Eq.(12):

$$
T_{j, k}^{h, B-U E}=\frac{1}{2} \log _{2}\left\{1+\frac{T p_{j, k}^{B, C R F C, r} \times C R_{p}^{r} \times h_{j, k}^{B, C R F C} \times h_{j, k}^{C R F C, U E}}{\left(N_{0} W\right) \times\left(T p_{j, k}^{B, C R F C, r} \times h_{j, k}^{B, C R F C}+C R_{p}^{r} \times h_{j, k}^{C R F C, U E}\right)}\right\}
$$

Similarly, the throughput achieved for the data transmis-

sion from UE to BS can be denoted as in Eq.(13):

$$
T_{j, k}^{h, U E-B}=\frac{1}{2} \log _{2}\left\{1+\frac{T p_{j, k}^{U E, C R F C, r} \times C R_{p, B S}^{r} \times h_{j, k}^{U E, C R F C} \times h_{k}^{C R F C, B S}}{\left(N_{0} W\right) \times\left(T p_{j, k}^{U E, C R F C, r} \times h_{j, k}^{U E, C R F C}+C R_{p, B S}^{r} \times h_{k}^{C R F C, B S}\right)}\right\}
$$

where $T p_{j, k}^{U E, C R F C}$ is the transmission power of the $j^{t h}$ UE transmitting data over the $k^{t h}$ subcarrier to the CR-FC, $h_{j, k}^{U E, C R F C}$ is the channel gain between UE and CR-FC, $h_{k}^{C R F C, B S}$ is the channel gain between CR-FC and BS.

A. Weighted Cooperative Spectrum Sensing and the Optimization Function

In this paper, the cooperative spectrum sensing scheme is considered in which energy samples from each CRs are fed to the CR-FC. The weight values are combined with the energy samples from each CRs. Therefore, the optimization problem here is to obtain the optimum weight values so as to maximize the probability of detection or minimize the probability of false alarm. For solving the optimization problem the Modified Whale Optimization Algorithm is employed. The optimal values of the fusion weights maximize the probability of correct detection for a specific value of the false alarm probability/minimize the probability of false alarm for a specific value of the probability of detection. The high detection probability and low false alarm probability implies that the spectrum sensing scheme can efficiently detect the presence and the absence of the Primary Users (PU) and can effectively provide the information about the vacant spectrum.

The mathematical model considered for spectrum sensing in this paper is as discussed below:

Spectrum Sensing for the transmission between $C R-F C$ and $B S$ :

$$
\begin{aligned}
& \mathrm{H}_{0}: y_{l}(t)=n_{l}(t) \\
& \mathrm{H}_{1}: y_{l}(t)=h_{l} s(t)+n_{l}(t) \\
& \{(\mathrm{PU} \text { Absent })\} \\
& \{(\text { PU Present })\}
\end{aligned}
$$

here, $y_{l}(t)$ : Signal sensed by the $l^{\text {th }}$ CRs at the $t^{t h}$ time instant. At each time instant an energy sample is sensed, the $t$ varies from 0 to $N-1$.

$h_{l}$ : Channel gain during the spectrum sensing performed by the $l^{\text {th }}$ CRs.

$s(t)$ : Licensed User/Primary User (PU) signal sensed at $t^{t h}$ time instant.

$n_{l}(t)$ : Zero mean AWGN for the $l^{t h}$ CRs at the $t^{t h}$ time instant with variance $\sigma_{l}^{2}$.

If $N$ is the number of spectrum sensing samples sensed by CRs, then the test statistics for each CRs can be written as in Eq.(15):

$$
U_{l}=\sum_{t=0}^{N-1}\left|y_{l}(t)\right|^{2}
$$

The statistics for the fusion center is: $\left\{z_{l}\right\}_{l=1}^{C R s}$, where $z_{l}=U_{l}+b_{l}, b_{l}$ is the noise induced by the control channel while transmission taking place between $l^{\text {th }}$ CRs and CRFC. The noise so induced is a AWGN with variance $v_{l}^{2}$. The fusion center calculates weighted value as in Eq.(16):

$$
\sum_{l=1}^{C R s} w_{l} z_{l}=\mathbf{w}^{T} \mathbf{z}
$$

where, $\mathbf{w}=\left[w_{1}, w_{2}, w_{3}, \ldots w_{C R s}\right]^{T}$

$\mathbf{z}=\left[z_{1}, z_{2}, z_{3}, \ldots . . z_{C R s}\right]^{T}$

$[.]^{T}$ :Transpose of the matrix.

With the above considered model and for a fixed probability of false alarm $P^{f}$, the probability of accurate detection can be evaluated as in Eq.(17) [8]:

$$
P^{d}=Q\left(\frac{Q^{-1}\left(P^{f}\right) \sqrt{\mathbf{w}^{T} \mathbf{C w}}-e_{s}[\mathbf{h}]^{T} \mathbf{w}}{\sqrt{\mathbf{w}^{T} \mathbf{D w}}}\right)
$$

where, $Q(x)=\frac{1}{\sqrt{2 \pi}} \int_{x}^{\infty} e^{-a^{2} / 2} d a, e_{s}=\sum_{t=0}^{N-1}|s(t)|^{2}$,

$\mathbf{h}=\left[\left|h_{1}\right|^{2},\left|h_{2}\right|^{2}, \ldots\left|h_{C R s}\right|^{2}\right]^{T}$

$\mathbf{C}=2 N \times \operatorname{diag}^{2}(\sigma)+\operatorname{diag}(v)$,

$\mathbf{D}=2 N \times \operatorname{diag}^{2}(\sigma)+\operatorname{diag}(v)$

$+4 e_{s} \times \operatorname{diag}(\mathbf{h})+\operatorname{diag}(v)$.

here diag(.) represents diagonal matrix.

$\sigma=\left[\sigma_{1}^{2}, \sigma_{2}^{2}, \ldots \ldots \sigma_{C R s}^{2}\right]^{T}, v=\left[v_{1}^{2}, v_{2}^{2}, \ldots v_{C R s}^{2}\right]^{T}$.

Similarly optimization function for minimizing the probability of false alarm can be written as in Eq.(18):

$$
P^{f}=Q\left(\frac{Q^{-1}\left(P^{d}\right) \sqrt{\mathbf{w}^{T} \mathbf{D} \mathbf{w}}}{\sqrt{\mathbf{w}^{T} \mathbf{C w}}-e_{s}[\mathbf{h}]^{T} \mathbf{w}}\right)
$$


Using Eq.(12), the achievable throughput for CR-FC for the data transmission from BS to UE can be written as in Eq.(19)[9]:

$$
T_{j, k}^{a c h, B-U E}=P^{H_{0}}\left(\frac{t^{f}-t^{s}}{t^{f}}\right)\left(1-P^{f}\right) T_{j, k}^{h, B-U E}
$$

here, $t^{f}$ frame duration of the selected vacant channel's frame structure employed for data transmission, $t^{s}$ is the spectrum sensing time and $P^{H_{0}}$ is the probability that $\mathrm{PU}$ is inactive.

Similarly, the achievable throughput for CR-FC for the data transmission from UE to BS can be written as in Eq.(20):

$$
T_{j, k}^{a c h, U E-B}=P^{H_{0}}\left(\frac{t^{f}-t^{s}}{t^{f}}\right)\left(1-P^{f}\right) T_{j, k}^{h, U E-B}
$$

The objective function for the optimization problem is to optimize $\mathbf{w}$ to maximize $P^{d}$ in Eq.(7) or to minimize $P^{f}$ in Eq.(8), such that $\sum_{l=1}^{C R s} w_{l}=1$, and $0 \leq w_{l} \leq 1$. In this paper, both the optimization problems i.e. maximizing $P^{d}$ and minimizing $P^{f}$ have been considered.

\section{EXPERT WhaLE OPTIMIZATION AlgORITHM}

\section{A. The Whale Optimization Algorithm}

The Whale Optimization Algorithm (WOA) developed in 2016 [10] is based on the hunting behavior of humpback whales. The WOA employs encircling mechanism, spiral position update technique and random search phase for finding an optimum solution.

Encircling Mechanism: In the encircling mechanism, the whales/search agents update their position as in Eq.(21) and Eq.(22) [10]:

$$
\begin{aligned}
& \overrightarrow{\mathrm{D}}=\left|\mathrm{C} \cdot X_{f, n}^{*}-X_{f, n}\right| \\
& X_{f, n+1}=X_{f, n}^{*}-\overrightarrow{\mathrm{A}} \cdot \overrightarrow{\mathrm{D}}
\end{aligned}
$$

here, $\overrightarrow{\mathrm{C}}$ is the coefficient vector and it is equal to $2 \cdot \vec{r}$, the best solution found so far in the current iteration $n$ $\left(n=0,1,2 \ldots n_{\max }\right)$ and at the $f^{t h}$ dimension $(f=0,1,2 \ldots d)$ is represented as $X_{f, n}^{*}$, search agents' position for the next iteration is represented by $\vec{X}_{f, n+1}, \overrightarrow{\mathrm{A}}$ is the discrimination weight coefficient and can be calculated as in Eq.(23):

$$
\overrightarrow{\mathrm{A}}=|2 \vec{a} \cdot \vec{r}-\vec{a}|
$$

here $\vec{a}$ is a linearly decreasing vector from 2 to $0, \vec{r}$ is a random vector having values between 0 and 1 .

The criteria for the encircling mechanism is that the $p \leq 0.5$ and $|A| \leq 1, p$ is random number between 0 and 1 .

The Figure 3 shows the pictorial representation of the encircling mechanism of the EWOA.

Spiral Position Update Technique: If $p>0.5$ then the search agent will move towards the prey/best solution in a spiral shape. The Figure 4 shows the 3D representation

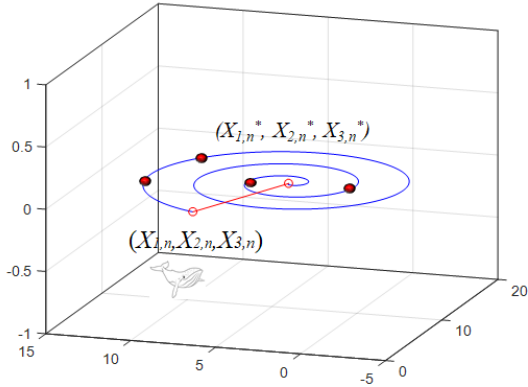

Figure 3: 3D representation of the Encircling position update of the search agent (Whale) towards the best solution

of the spiral position updates of the whales towards the best solution $\left(X_{1, n}^{*}, X_{2, n}^{*}, X_{3, n}^{*}\right)$ of a 3 dimensional problem. The equation governing this technique is as in Eq.(24) and Eq.(25):

$$
\begin{gathered}
X_{f, n+1}=\vec{D} \cdot e^{b l} \cdot \cos (2 \pi l)+X_{f, n}^{*}, \quad l \in[-1,1] \\
\overrightarrow{\mathrm{D}}=\left|X_{f, n}^{*}-X_{f, n}\right|
\end{gathered}
$$

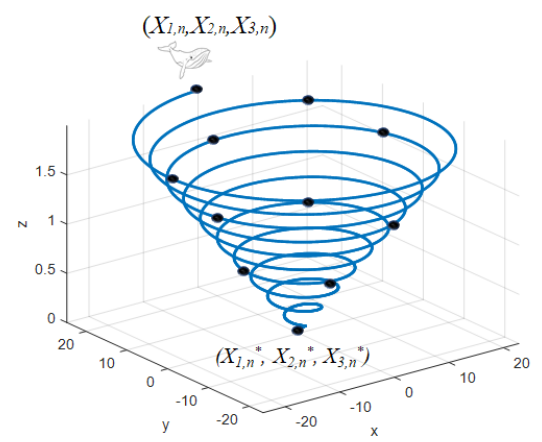

Figure 4: 3D representation of the spiral position update of the search agent (Whale) towards the best solution

Random Search Phase: This phase is initiated so that optimization algorithm performs good exploration and have global search.

The condition for random global search is that the $p \leq 0.5$ and $|A|>1$. In this phase, instead of searching around the best solution $X_{n}^{*}$, the algorithm randomly selects a solution $X_{f, n}^{r a n d}$ and search agents move towards it as in Eq.(26) and Eq.(27):

$$
\begin{aligned}
& \overrightarrow{\mathrm{D}}=\left|\overrightarrow{\mathrm{C}} \cdot X_{f, n}^{\text {rand }}-X_{f, n}\right| \\
& X_{f, n+1}=X_{f, n}^{r a n d}-\mathrm{A} \cdot \overrightarrow{\mathrm{D}}
\end{aligned}
$$




\section{B. Expert balance between Exploration and Exploitation}

For an optimization algorithm it is important to have proper tradeoff between exploration and exploitation abilities [12]. In WOA, exploitation is well carried out, but for exploration random solution is picked irrespective of its position from the best solution. This randomly chosen solution can also be near to the already exploited region. In order to have better global search so as to overcome local optima, a threshold is introduced for choosing a solution as random solution. The solutions which are far from the best solutions i.e. solutions which are in unexplored region is preferred for initializing the global search.

Modification can be mathematically modeled as in equations (28 to 31$)$ :

$$
\begin{aligned}
& d_{1}=\sqrt{X_{f, n}^{*}-X_{f, n}^{\text {rand }}} \\
& d_{2}=\sqrt{X_{f, n}^{*}-X_{f, n}^{\text {best }}}
\end{aligned}
$$

where, $X_{f, n}^{b_{f} t_{2}}$ is the 2 nd best solution among all the search agents.

if $d_{1}>d_{2}$, and $p \leq 0.5$ and $|A|>1$ then update search agents as:

$$
\begin{aligned}
& \overrightarrow{\mathrm{D}}=\left|\overrightarrow{\mathrm{C}} \cdot X_{f, n}^{r \text { rand }}-X_{f, n}\right| \\
& X_{f, n+1}=X_{f, n}^{r a n d}-\overrightarrow{\mathrm{A}} \cdot \overrightarrow{\mathrm{D}}
\end{aligned}
$$

The proposed modification via Eqs.(28-31) can be termed as Modified Random Search Phase.

For an optimization algorithm it is important that its search agents reach almost all possible solution in the search space without slowing down the entire search process [19]. So, in this paper Mutation and Replacement strategy is applied. In each iteration, the search agents undergoes mutation and the corresponding solution then replaces the worst solution in the solution set to make sure proper that the search agents overcome local optima and progresses towards global best solution.

After Encircling Mechanism / Spiral Update Mechanism and Modified Random Search Phase, the search agents undergoes Polynomial Mutation Scheme, in which offspring is generated based on Eq. 32:

$$
X_{f, n}^{m u t}=X_{f, n}+\left(X_{f, n}^{U}-X_{f, n}^{L}\right) \delta_{f, n}
$$

here, $X_{f, n}^{U}$ and $X_{f, n}^{L}$ are the upper and lower bounds of $X_{f, n}$. The $\delta_{f, n}$ is computed via polynomial probability distribution:

$$
\begin{gathered}
P(\delta)=0.5\left(\eta^{m}+1\right)\left(1-|\delta|^{\eta^{m}}\right) \\
\delta_{f, n}=\left\{\begin{array}{c}
2 r_{f, n}^{\frac{1}{\left.\eta^{m}+1\right)}}-1, \quad r_{f, n}<0.5 \\
1-2\left(1-r_{f, n}\right)^{\frac{1}{\left.\eta^{m}+1\right)}}, \text { otherwise }
\end{array}\right.
\end{gathered}
$$

where, $\eta^{m}$ is generally $\approx 20$ and $r_{f, n}$ is random number ranging in $[0,1]$. Based on fitness of the solutions the search agents are categorized and the worst solution is replaced by the mutated solution $\vec{X}_{f, n}^{m u t}$. In this way, the entire search agents would be led towards the global optimum solution. The proposed EWOA has efficient exploration scheme with which random search agent are selected such a way as shown in equations (28-31) that it overcomes local optima. With polynomial mutation scheme as described in equations (3234) the proposed EWOA has double edge sword of efficient tradeoff between exploration and exploitation therefore it converge towards the global optimum solution as shown in Figures 3 and 4.

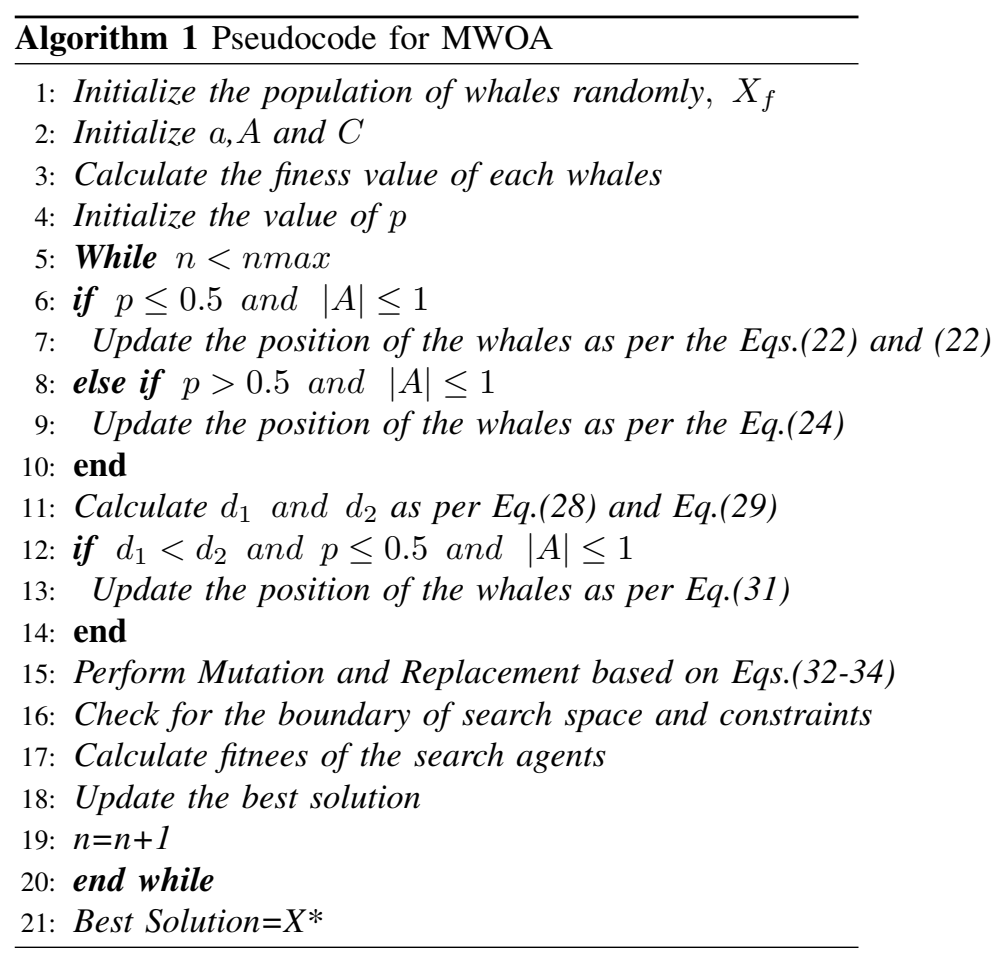

\section{System Model of the Cooperative Cognitive RADIO NETWORK}

The Figure 5 shows the system model of the cooperative cognitive radio network considered. The EWOA is employed for the optimizing the weights in the fusion center as shown in Figure 5. The weights $\mathbf{w}=\left[w_{1}, w_{2}, w_{3}, \ldots . w_{C R s}\right]^{T}$ employed in the Fusion Center are efficiently optimized by the proposed EWOA.

\section{Simulation Results}

In this paper, attempt is made to separately optimize the weights in Eq.(17) and Eq.(18) so as to minimize probability of false alarm and to maximize probability of detection. An efficient spectrum sensing algorithm is identified by its high probability of detection and low probability of false alarm. The proposed MWOA based spectrum sensing is compared with PSO, WOA and conventional energy detector based spectrum sensing. The comparison is made in terms 


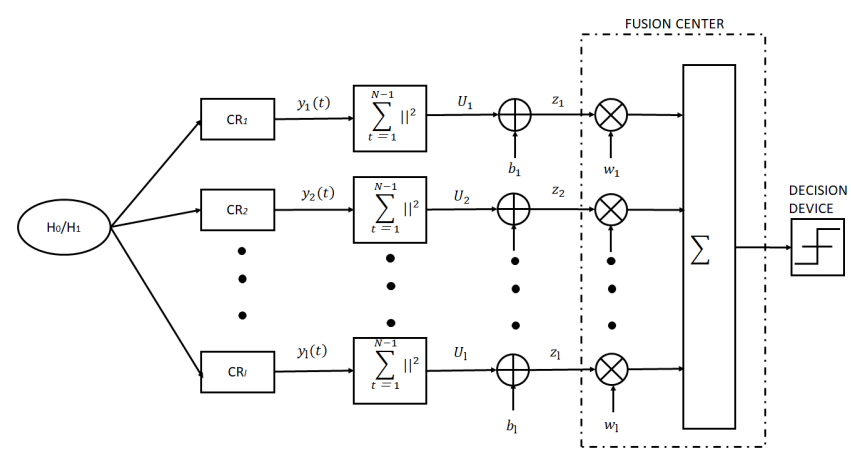

Figure 5: System Model of the Cooperative Cognitive Radio Network [34]

of convergence curve for $P^{f}$ and $P^{d}$, and the average throughput for the relay based scenario. The parameters considered for simulation are: The number of $C R_{s}=5$, the AWGN variance is $\sigma=[0.06,0.06,0.06,0.06,0.06]^{T}$, control channel noise variance $v=[0.06,0.06,0.06,0.06,0.06]^{T}$, $W=60 \mathrm{KHz}, \quad N_{0}=1 \times 10^{-6} \mathrm{~W} / \mathrm{Hz}$. The base station transmission power ranges from $25 \mathrm{dBm}$ to $45 \mathrm{dBm}$, CR-FC transmission power ranges from $20 \mathrm{dBm}$ to $30 \mathrm{dBm}$ [11]. The UE transmission power is set to $0.1 \mathrm{~W}$. The channel gains $h_{j, k, t}^{B, C R F C}, h_{j, k, t}^{C R C, U E}$ and $h_{j, k, t}^{U E, C R F C}, h_{j, k, t}^{C R F C, B}$ are considered to be Rayleigh faded channel. The channel between LU and CRs is considered to be independent of the channels $h_{j, k, t}^{B, C R F C}, h_{j, k, t}^{C R F C, U E}$ and $h_{j, k, t}^{U E, C R F C}, h_{j, k, t}^{C R F C, B}$. The $\alpha=11.9, \quad \beta=0.14, \quad J_{1}=10.39, \quad J_{2}=0.05, \quad G_{1}=29.06$, $G_{2}=0.03, d_{o}$ reference distance $=1 \mathrm{~m}, \Upsilon_{1}=3 \mathrm{~dB}, \Upsilon_{2}=23 \mathrm{~dB}$ [20]. For optimizing $P^{f}$, the $P^{d}$ is considered to be 0.9 and for optimizing $P^{d}$, the $P^{f}$ is taken as 0.1 and $P^{H_{0}}$ is considered 0.8. The number of search agents/whales/particles considered are 50 for MWOA,WOA and PSO. Each optimization algorithm run 30 times and each run is equal to the 500 iterations.

Table I: Simulation Parameter values

\begin{tabular}{cc}
\hline Parameter & Values \\
\hline$\alpha$ & 11.9 \\
$\beta$ & 0.14 \\
$J_{1}$ & 10.39 \\
$J_{2}$ & 0.05 \\
$G_{1}$ & 29.06 \\
$G_{2}$ & 0.03 \\
$d_{o}$ reference distance & $1 \mathrm{~m}$ \\
$\Upsilon_{1}$ & $3 \mathrm{~dB}$ \\
$\Upsilon_{2}$ & $23 \mathrm{~dB}$ \\
$h$ & $30 \mathrm{~m}$ \\
$h_{B S}$ & $20 \mathrm{~m}$ \\
\hline \hline
\end{tabular}

The Figure 6 and Figure 7 shows the comparison between optimization algorithms (EWOA, WOA and PSO) to efficiently optimize the weights in equations (6) and (7) so as to maximize $P^{d}$ and minimize $P^{f}$. This is because the pro-

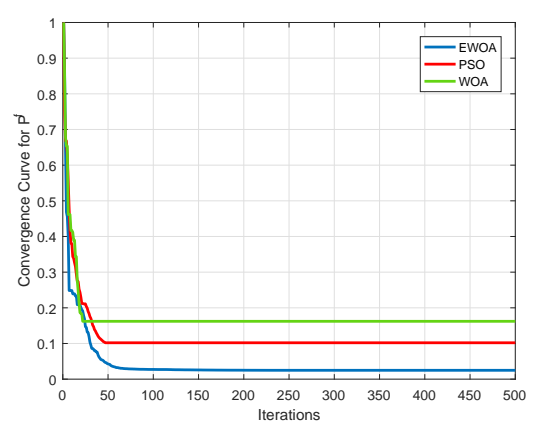

Figure 6: Convergence Curve of Probability of False Alarm

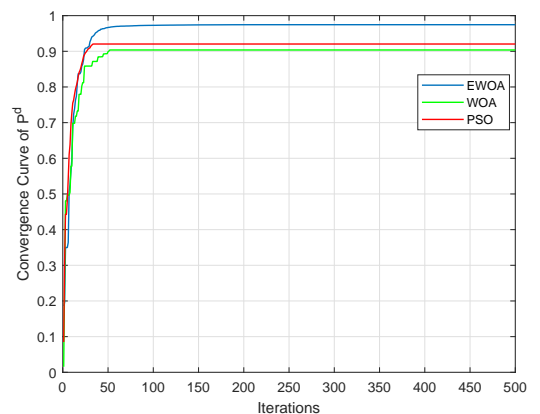

Figure 7: Convergence Curve of Probability of Detection

posed EWOA is successful in maintaining the efficient balance between its exploration and exploitation abilities. The EWOA has good approach of avoiding local optima solution by effectively mutating the search agents and replacing the worst solution. In this way the algorithm move towards the global best solution. From the convergence curve it can be inferred that for the 500 iterations EWOA has successfully outperformed WOA and PSO to obtain $P^{d}$ value of 0.97 and $P^{f}$ value 0.02 . The WOA and PSO has obtained $P^{d}$ value as 0.90 and 0.92 respectively and for $P^{f}$ the values for WOA and PSO are 0.1 and 0.18 respectively. The EWOA has $7.7 \%$ and $5.4 \%$ better performance compared to WOA and PSO in terms of convergence for Probability of detection. For the convergence of probability of false, the proposed EWOA has $80 \%$ and $88 \%$ better performance as compared to PSO and WOA respectively. The better values of $P^{f}$ so obtained using EWOA is clearly reflected in the average throughput so obtained for BS to UE and UE to BS transmission using CR based relay under network blockage area as shown in Figures 8 and 9. For the sensing time of $3 \mathrm{~ms}$ and frame period 50 , the average throughput obtained for varying SNR values is higher for proposed MWOA based spectrum sensing as compared to WOA, PSO and conventional energy detection based spectrum sensing.

The optimized weights obtained via proposed EWOA is employed to obtain receiver operating characteristics (ROC) for different values of probability of detection and prob- 


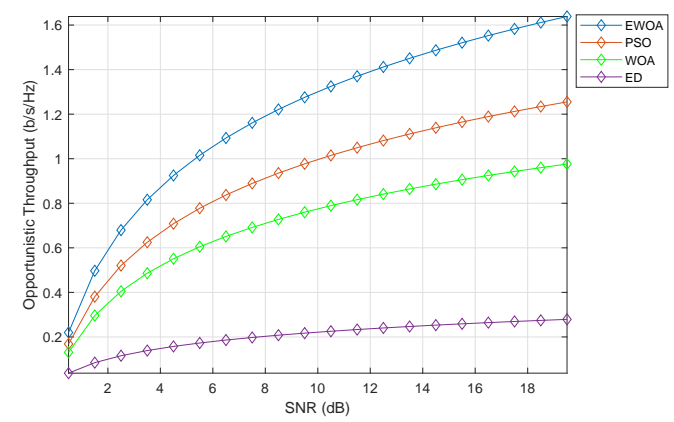

Figure 8: Opportunistic Throughput vs SNR (For UE to BS)

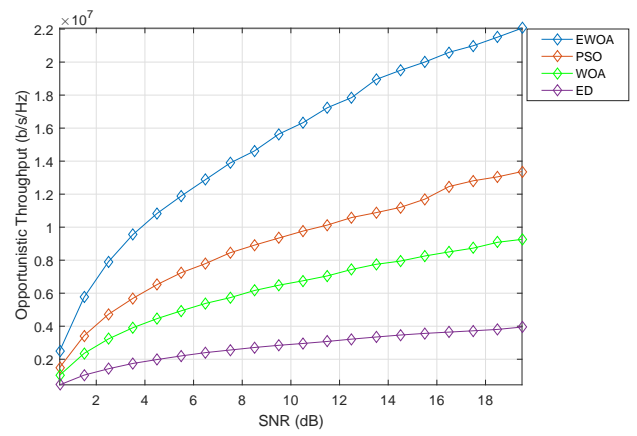

Figure 9: Opportunistic Throughput vs SNR (For BS to UE)

ability of false alarm as shown in Figure 10. The ROC for EWOA is compared with the PSO, WOA, and Energy Detector (ED).

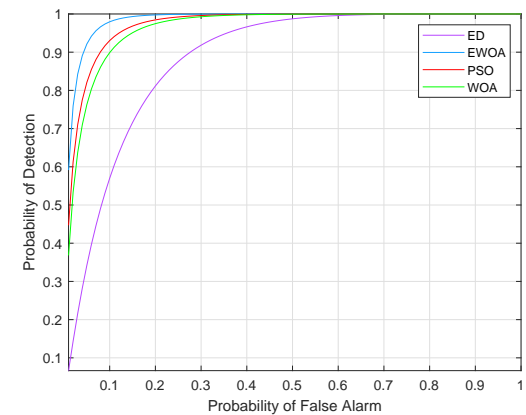

Figure 10: Receiver Operating Characteristics

From Figure 10, it can be inferred that even for the low probability of false alarm, the detection probability of MWOA based Cognitive radio network is better as compared to PSO, WOA and ED. The performance of EWOA in effectively detecting the spectrum holes is justified by the BER performance as shown in Figure 11. The efficient mutation and expert exploration scheme has efficiently enhanced the performance of the EWOA and as result the presence and the

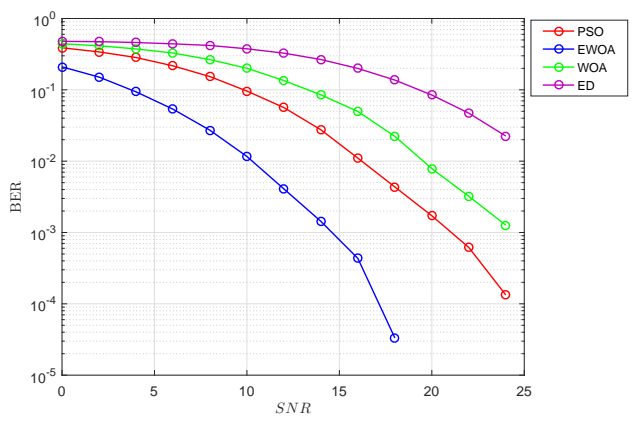

Figure 11: BER vs SNR

absence of the PU is detected with high detection probability and low false alarm probability.

\section{CONCLUSION}

In this paper, to mitigate the problem of network blockage and to enhance the data connection for the wireless PMS, the cognitive radio based relay scheme is proposed to improve the network performance of the user equipment in the network blockage regions. Moreover, the modified whale optimization algorithm is employed for spectrum sensing optimization to increase the efficiency of the cognitive radio network. From the simulation results, it is proved that EWOA optimized spectrum sensing has significantly improved the performance of the cognitive radio network by efficiently detecting the vacant spectrum. The network performance with respect to opportunistic throughput from UE to BS of EWOA based cognitive radio network has increased by $33 \%$ and $60 \%$ as compared to existing PSO and WOA based system respectively. Whereas, the opportunistic throughput achieved for data transmission from BS to UE has increased by $57 \%$ and $120 \%$ as compared to PSO and WOA respectively. Therefore, with EWOA optimized spectrum sensing a wireless cognitive radio relay based PMS can efficiently detect the spectrum holes even in network blockage condition. Thus, it can provide seamless connection for efficient wireless remote PMS.

\section{ACKNOWLEDGMENT}

The authors would like to acknowledge CSIR (Council of Scientific and Industrial Research) for their support under SRF (Senior Research Fellowship) Program at VIT Vellore and U.K. Commonwealth for their support as Split-Site scholarship Program in London.

\section{CONFLICT OF INTEREST}

The authors declare that they have no conflict of interest.

\section{REFERENCES}

[1] Choi, Y., Ji, H. W., Park, J. Y., Kim, H. C., and Silvester, J. A. (2011). A $3 \mathrm{~W}$ network strategy for mobile data traffic offloading. IEEE Communications Magazine, 49(10), 118-123. 
[2] Rosnes, E., and Ytrehus, Y. (2005). Improved algorithms for the determination of turbo-code weight distributions. IEEE Transactions on communications, 53(1), 20-26.

[3] Naguib, A. F., Tarokh, V., Seshadri, N., and Calderbank, A. R. (1998). A space-time coding modem for high-data-rate wireless communications. IEEE Journal on Selected areas in Communications, 16(8), 1459-1478.

[4] Tarokh, V., Seshadri, N., and Calderbank, A. R. (1998). Spacetime codes for high data rate wireless communication: Performance criterion and code construction. IEEE transactions on information theory, 44(2), 744-765.

[5] Xiang, W., Zheng, K., and Shen, X. S. (Eds.). (2016). 5G mobile communications. Springer.

[6] Yucek, T., and Arslan, H. (2009). A survey of spectrum sensing algorithms for cognitive radio applications. IEEE communications surveys and tutorials, 11(1), 116-130.

[7] Berger, S., Kuhn, M., Wittneben, A., Unger, T., and Klein, A. (2009). Recent advances in amplify-and-forward two-hop relaying. IEEE Communications Magazine, 47(7), 50-56.

[8] Quan, Z., Cui, S., and Sayed, A. H. (2008). Optimal linear cooperation for spectrum sensing in cognitive radio networks. IEEE Journal of selected topics in signal processing, 2(1), 2840.

[9] Rashid, R. A., Hamid, A. H. F. B. A., Fisal, N., SyedYusof, S. K., Hosseini, H., Lo, A., and Farzamnia, A. (2015). Efficient in-band spectrum sensing using swarm intelligence for cognitive radio network. Canadian Journal of Electrical and Computer Engineering, 38(2), 106-115.

[10] Mirjalili, S., and Lewis, A. (2016). The whale optimization algorithm. Advances in engineering software, 95, 51-67.

[11] Dahlman, E., Parkvall, S., Skold, J., and Beming, P. (2010). $3 \mathrm{G}$ evolution: HSPA and LTE for mobile broadband. Academic press.

[12] Eappen, G., and Shankar, T. (2020). Hybrid PSO-GSA for energy efficient spectrum sensing in cognitive radio network. Physical Communication, 101091.

[13] Venugopal, K., Valenti, M. C., and Heath, R. W. (2016). Device-to-device millimeter wave communications: Interference, coverage, rate, and finite topologies. IEEE Transactions on Wireless Communications, 15(9), 6175-6188.

[14] Balanis, C. A. (2016). Antenna theory: analysis and design. John wiley and sons.

[15] Mondal, B., Thomas, T. A., Visotsky, E., Vook, F. W., Ghosh, A., Nam, Y. H., ... and Kakishima, Y. (2015). 3D channel model in 3GPP. IEEE Communications Magazine, 53(3), 16-23.

[16] Al-Hourani, A., Kandeepan, S., and Jamalipour, A. (2014, December). Modeling air-to-ground path loss for low altitude platforms in urban environments. In 2014 IEEE global communications conference (pp. 2898-2904). IEEE.
[17] Mozaffari, M., Saad, W., Bennis, M., Nam, Y. H., and Debbah, M. (2019). A tutorial on UAVs for wireless networks: Applications, challenges, and open problems. IEEE communications surveys and tutorials, 21(3), 2334-2360.

[18] Mozaffari, M., Saad, W., Bennis, M., and Debbah, M. (2016). Unmanned aerial vehicle with underlaid device-to-device communications: Performance and tradeoffs. IEEE Transactions on Wireless Communications, 15(6), 3949-3963.

[19] Talbi, E. G. (2009). Metaheuristics: from design to implementation (Vol. 74). John Wiley and Sons.

[20] Mozaffari, M. (2018). Wireless Communications and Networking with Unmanned Aerial Vehicles: Fundamentals, Deployment, and Optimization (Doctoral dissertation, Virginia Tech).

[21] Lin, X., Andrews, J. G., Ghosh, A., and Ratasuk, R. (2014). An overview of 3GPP device-to-device proximity services. IEEE Communications Magazine, 52(4), 40-48.

[22] 3GPP,3rd Generation Partnership Project; Technical Specification Group SA; Feasibility Study for Proximity Services (ProSe) (Release 12). TR 22.803 V1.0.0, August 2012

[23] Song, L., Niyato, D., Han, Z., and Hossain, E. (2015). Wireless device-to-device communications and networks. Cambridge University Press.

[24] Choi, K. W., and Han, Z. (2014). Device-to-device discovery for proximity-based service in LTE-advanced system. IEEE Journal on Selected Areas in Communications, 33(1), 55-66.

[25] Lei, L., Zhong, Z., Lin, C., and Shen, X. (2012). Operator controlled device-to-device communications in LTE-advanced networks. IEEE Wireless Communications, 19(3), 96-104.

[26] Song, L., Niyato, D., Han, Z., and Hossain, E. (2014). Game-theoretic resource allocation methods for device-todevice communication. IEEE Wireless Communications, 21(3), 136-144.

[27] Tehrani, M. N., Uysal, M., and Yanikomeroglu, H. (2014). Device-to-device communication in $5 \mathrm{G}$ cellular networks: challenges, solutions, and future directions. IEEE Communications Magazine, 52(5), 86-92.

[28] Asadi, A., Wang, Q., and Mancuso, V. (2014). A survey on device-to-device communication in cellular networks. IEEE Communications Surveys and Tutorials, 16(4), 1801-1819.

[29] Li, P., and Guo, S. (2014). Cooperative device-to-device communication in cognitive radio cellular networks. Springer International Publishing.

[30] Laneman, J. N., Tse, D. N., and Wornell, G. W. (2004). Cooperative diversity in wireless networks: Efficient protocols and outage behavior. IEEE Transactions on Information theory, 50(12), 3062-3080.

[31] Sultana, A., Zhao, L., and Fernando, X. (2017). Efficient resource allocation in device-to-device communication using cognitive radio technology. IEEE Transactions on Vehicular Technology, 66(11), 10024-10034. 
[32] Gong, X., Yuan, W., Liu, W., Cheng, W., and Wang, S. (2008, November). A cooperative relay scheme for secondary communication in cognitive radio networks. In IEEE GLOBECOM 2008-2008 IEEE Global Telecommunications Conference (pp. 1-6). IEEE.

[33] He, C., Feng, Z., Zhang, Q., Zhang, Z., and Xiao, H. (2010, September). A joint relay selection, spectrum allocation and rate control scheme in relay-assisted cognitive radio system. In 2010 IEEE 72nd Vehicular Technology Conference-Fall (pp. 1-5). IEEE.

[34] Zheng, S., Lou, C., and Yang, X. (2010). Cooperative spectrum sensing using particle swarm optimisation. Electronics letters, 46(22), 1525-1526.

[35] Rashid, R. A., Hamid, A. H. F. B. A., Fisal, N., SyedYusof, S. K., Hosseini, H., Lo, A., and Farzamnia, A. (2015). Efficient in-band spectrum sensing using swarm intelligence for cognitive radio network. Canadian Journal of Electrical and Computer Engineering, 38(2), 106-115.

[36] Das, D., and Das, S. (2014, February). A cooperative spectrum sensing scheme using multiobjective hybrid IWO/PSO algorithm in cognitive radio networks. In 2014 International Conference on Issues and Challenges in Intelligent Computing Techniques (ICICT) (pp. 225-230). IEEE.

[37] Eappen, G., and Shankar, T. (2020). Hybrid PSO-GSA for energy efficient spectrum sensing in cognitive radio network. Physical Communication, 101091.

[38] Yang, X. S. (2014). Swarm intelligence based algorithms: a critical analysis. Evolutionary intelligence, 7(1), 17-28. 